\title{
The role of Galactic unresolved PWNe to the gamma-ray diffuse emission
}

\author{
G. Pagliaroli, ${ }^{a, b, *}$ V. Vecchiotti ${ }^{a, b}$ and F.L. Villante ${ }^{b, c}$ \\ ${ }^{a}$ Gran Sasso Science Institute, 67100 L'Aquila, Italy \\ ${ }^{b}$ INFN, Laboratori Nazionali del Gran Sasso, 67100 Assergi (AQ), Italy \\ ${ }^{c}$ University of L'Aquila, Physics and Chemistry Department, 67100 L'Aquila, Italy \\ E-mail: giulia.pagliaroli@gssi.it
}

\begin{abstract}
We discuss the implications of the TeV galactic Pulsar Wind Nebulae (PWNe) population observed by HESS Galactic Plane Survey (HGPS) for the interpretation of Fermi-LAT data in the GeV domain. We show that consistency among HGPS and 3FGL catalogs requires that the average ratio $R_{\Phi}=\Phi_{\mathrm{GeV}} / \Phi_{\mathrm{TeV}}$, where $\Phi_{\mathrm{GeV}}\left(\Phi_{\mathrm{TeV}}\right)$ is the integrated gamma-ray flux emitted by sources in the range $1-100 \mathrm{GeV}(1-100 \mathrm{TeV})$ probed by Fermi-LAT (HESS), is $R_{\Phi}=500-1000$. Considering that the average spectral index of observed sources at $\mathrm{TeV}$ energies is $\beta_{\mathrm{TeV}}=2.3$, the required value for $R_{\Phi}$ can be only obtained by assuming that sources have spectral break below $1 \mathrm{TeV}$ and harder emission spectrum with $\beta_{\mathrm{GeV}}<2$ in the GeV domain. Such spectral shapes are well compatible with the expected gamma-ray emission in young PWNe due to Inverse Compton scattering of high-energy electrons on background radiation fields. Finally, we show that a relevant fraction of the $\mathrm{TeV}$ source population cannot be resolved by Fermi-LAT in the $\mathrm{GeV}$ domain. We suggest that unresolved sources can provide a not negligible contribution to the large-scale diffuse emission observed by Fermi-LAT, possibly explaining the spectral hardening of this component toward the galactic center reported by Gaggero et al. 2018, Yang et al. 2016, Acero et al. 2016.
\end{abstract}

$37^{\text {th }}$ International Cosmic Ray Conference (ICRC 2021)

July 12th-23rd, 2021

Online - Berlin, Germany

\footnotetext{
*Presenter
} 


\section{Introduction}

In the last decade, Imaging Atmospheric Cherenkov Telescopes (IACT), like H.E.S.S., MAGIC and VERITAS, and air shower arrays, such as Argo-YBJ, Milagro and HAWC, provided a detailed description of Galactic $\gamma$-ray emission in the energy range $0.1-100 \mathrm{TeV}$. The emerging picture is that $\mathrm{TeV}$ Galactic sky is dominated by a population of bright sources powered by pulsar activity, such as pulsar wind nebulae (PWNe) [1] or TeV halos [9], whose properties can be effectively constrained by observations at TeV energies, see e.g. [5] These objects are expected to emit also in the $\mathrm{GeV}$ energy domain where, however, population studies are more difficult because different kinds of sources dominate the emission.

In this paper, as also discussed in [11], we took advantage of the constraints provided by H.E.S.S. Galactic Plane Survey (HGPS) to discuss the implications of TeV PWNe for the interpretation of Fermi-LAT data in the GeV domain. We quantify the contribution of unresolved TeV PWNe to large scale diffuse emission observed by Fermi-LAT at different distances from the Galactic center. We show that the inclusion of this additional component can strongly affect the CR energy distribution reconstructed from Fermi-LAT data, weakening the evidence of a progressive hardening of the cosmic-ray spectrum toward the Galactic center.

\section{Method}

Source average spectrum. We consider the hypothesis, suggested e.g. by [9], that most of the bright $\mathrm{TeV}$ sources in the Galaxy are young PWNe and/or TeV halos. In this scenario, the observed $\gamma$-ray emission is produced by IC scattering of HE electron and positrons on background photons (CMB, starlight, infrared). At GeV energies, this naturally produces hard $\gamma$-ray emission, since the scattered photon spectral index in the Thompson regime is $\beta \sim(p+1) / 2$, where $p$ is the spectral index of injected electrons/positrons. At TeV energy, a softer $\gamma$-ray spectrum is produced either due to the Klein-Nishina regime in which $\beta \sim(p+1)$ and/or to electron/positron energy losses, see e.g.[10]. We take this into account by parameterizing the source emission with a broken power-law with different spectral indexes $\beta_{\mathrm{GeV}}$ and $\beta_{\mathrm{TeV}}$ in the $\mathrm{GeV}$ and $\mathrm{TeV}$ energy domain and with a transition energy $E_{0}=0.3 \mathrm{TeV}$ located between the ranges probed by Fermi-LAT and HESS.

At high energies ( $\left.E \geq E_{0}\right)$, we take the average spectrum observed by HESS [2] as a reference, i.e. we assume that all sources have $\beta_{\mathrm{TeV}}=2.3$. The index $\beta_{\mathrm{GeV}}$ is instead determined by requiring that the ratio

$$
R_{\Phi} \equiv \Phi_{\mathrm{GeV}} / \Phi_{\mathrm{TeV}}
$$

between the integrated fluxes $\Phi_{\mathrm{GeV}}$ and $\Phi_{\mathrm{TeV}}$ emitted by a given source in the $\mathrm{GeV}$ and TeV energy domains, is consistent with observations. This quantity can be expressed as a function of $\beta_{\mathrm{GeV}}$ and $\beta_{\mathrm{TeV}}$, obtaining:

$$
R_{\Phi}=\frac{1-\beta_{\mathrm{TeV}}}{1-\beta_{\mathrm{GeV}}} \frac{\left[\left(\epsilon_{\mathrm{GeV}}^{\mathrm{sup}}\right)^{1-\beta_{\mathrm{GeV}}}-\left(\epsilon_{\mathrm{GeV}}^{\mathrm{inf}}\right)^{1-\beta_{\mathrm{GeV}}}\right]}{\left[\left(\epsilon_{\mathrm{TeV}}^{\mathrm{sup}}\right)^{1-\beta_{\mathrm{TeV}}}-\left(\epsilon_{\mathrm{TeV}}^{\mathrm{inf}}\right)^{1-\beta_{\mathrm{TeV}}}\right]}
$$

where $\epsilon_{\mathrm{GeV}}^{\mathrm{inf}} \equiv\left(1.0 \mathrm{GeV} / E_{0}\right)$ and $\epsilon_{\mathrm{GeV}}^{\text {sup }} \equiv\left(100 \mathrm{GeV} / E_{0}\right)\left(\epsilon_{\mathrm{TeV}}^{\mathrm{inf}} \equiv\left(1.0 \mathrm{TeV} / E_{0}\right)\right.$ and $\epsilon_{\mathrm{TeV}}^{\text {sup }} \equiv$ $\left.\left(100 \mathrm{TeV} / E_{0}\right)\right)$ are the lower and upper bounds of the $\mathrm{GeV}(\mathrm{TeV})$ energy domains. Realistic 
values for $R_{\Phi}$ can be obtained from observations by considering the ensemble of PWNe that are firmly identified both in the 3FGL and HGPS catalogs (6 objects). By taking the ratio of the integrated fluxes $\Phi_{\mathrm{GeV}}$ and $\Phi_{\mathrm{TeV}}$ measured by Fermi-LAT and H.E.S.S., we obtain $R_{\Phi} \leq 1200$ (with an average value $R_{\Phi} \simeq 700$ ). In our calculations, we take as a reference the values $R_{\Phi}=500$ and 1000 that correspond to $\beta_{\mathrm{GeV}} \simeq 1.7$ and 1.9 , respectively.

Source population. The source space and luminosity distribution is described by:

$$
\frac{d N}{d^{3} r d L_{\mathrm{TeV}}}=\rho(\mathbf{r}) Y_{\mathrm{TeV}}\left(L_{\mathrm{TeV}}\right)
$$

where $r$ indicates the distance from the Galactic Center. The function $\rho(\mathbf{r})$ is conventionally normalized to one when integrated in the entire Galaxy. It is assumed to be proportional to the pulsar distribution parameterized by [7] and to scale as $\exp (-|z| / H)$ with $H=0.2 \mathrm{kpc}$, along the direction $z$ perpendicular to the Galactic plane. The function $Y_{\mathrm{TeV}}\left(L_{\mathrm{TeV}}\right)$ gives the source intrinsic luminosity distribution in the $\mathrm{TeV}$ energy domain. It is parameterized as a power-law:

$$
Y_{\mathrm{TeV}}\left(L_{\mathrm{TeV}}\right)=\frac{R \tau(\alpha-1)}{L_{\mathrm{TeV}, \operatorname{Max}}}\left(\frac{L_{\mathrm{TeV}}}{L_{\mathrm{TeV}, \mathrm{Max}}}\right)^{-\alpha}
$$

in the luminosity range $L_{\mathrm{TeV}}$, Min $\leq L_{\mathrm{TeV}} \leq L_{\mathrm{TeV}}$, Max. This distribution is naturally obtained for a population of fading sources, such as PWNe or TeV Halos, created at a constant rate $R$ and having intrinsic luminosity that decreases over a time scale $\tau$ according to:

$$
L_{\mathrm{TeV}}(t)=L_{\mathrm{TeV}, \operatorname{Max}}\left(1+\frac{t}{\tau}\right)^{-\gamma}
$$

where $t$ indicates the time passed since source formation. In this assumption, the exponent $\alpha$ of the luminosity distribution is given by $\alpha=1 / \gamma+1$.

The birth rate of PWNe or TeV Halos is similar to that of SN explosions in our Galaxy, i.e. $R \simeq R_{\mathrm{SN}}=0.019 \mathrm{yr}^{-1}[6]$. Since $\gamma$-ray emission is powered by pulsar activity, their TeV-luminosity can be connected to the pulsar spin-down power, i.e.:

$$
L_{\mathrm{TeV}}=\lambda \dot{E}
$$

where $\lambda \leq 1$ and

$$
\dot{E}=\dot{E}_{0}\left(1+\frac{t}{\tau_{\mathrm{sd}}}\right)^{-2}
$$

for energy loss dominated by magnetic dipole radiation (braking index $n=3$ ). This implies that the fading timescale is determined by the pulsar spin-down time scale, i.e. $\tau=\tau_{\mathrm{sd}}$. If the efficiency of $\mathrm{TeV}$ emission does not depend on time ( $\lambda \sim$ const), the exponent in Eq. (5) is $\gamma=2$, that corresponds to a source luminosity function $Y_{\mathrm{TeV}}\left(L_{\mathrm{TeV}}\right) \propto L_{\mathrm{TeV}}^{-1.5}$. The possibility of $\lambda$ being correlated to the spin-down power, i.e. $\lambda=\lambda_{0}\left(\dot{E} / \dot{E}_{0}\right)^{\delta}$, was suggested by [1] that found $L_{\mathrm{TeV}}=\lambda \dot{E} \propto \dot{E}^{1+\delta}$ with $1+\delta=0.59 \pm 0.21$ by studying a sample of PWNe in the HPGS catalogue. In this case, one obtains $\gamma \simeq 1.2$ in Eq. (5) that corresponds to a source luminosity function $Y_{\mathrm{TeV}}\left(L_{\mathrm{TeV}}\right) \propto L_{\mathrm{TeV}}^{-1.8}$. These two scenarios ( $\alpha=1.5$ and $\alpha=1.8)$ are considered as working hypotheses for our work. 


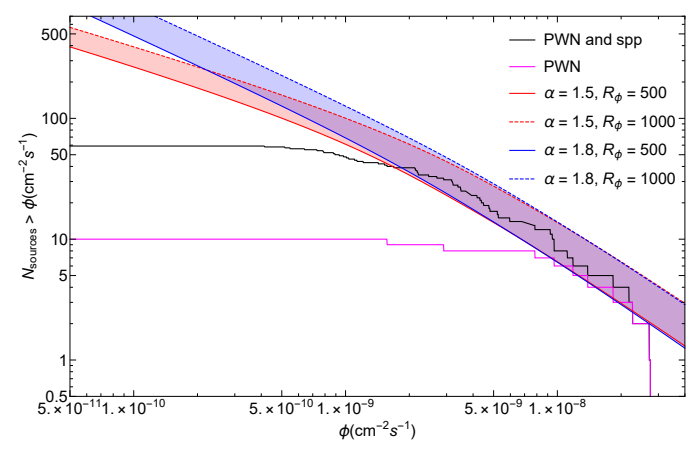

Figure 1: The cumulative number $N\left(\Phi_{\mathrm{GeV}}\right)$ of sources with fluxes larger than $\Phi_{\mathrm{GeV}}$. See text for details.

Consistency among HGPS and Fermi-LAT catalogs. In a recent paper [5], we have shown that the parameters $L_{\mathrm{TeV}}$, Max and $\tau$ are constrained by the flux, latitude and longitude distributions of bright TeV-sources observed by HGPS. The implications of the considered population at $\mathrm{GeV}$ energies also depend on the parameter $R_{\Phi}$ which is related to the assumed source spectrum. The reference values $R_{\Phi}=500$ and $R_{\Phi}=1000$ can be further validated by comparing the predicted source flux distribution in the GeV domain with the results of the Fermi-LAT 3FGL catalogue. The two shaded bands in Fig. 1 show the cumulative number $N\left(\Phi_{\mathrm{GeV}}\right)$ of expected sources in the latitude range $|b| \leq 20.25^{\circ}$ with flux larger than $\Phi_{\mathrm{GeV}}$ for two different values of the power-law index of the luminosity function ( $\alpha=1.5$ and 1.8). Namely, the red (blue) shaded band is obtained by assuming the best-fit values $L_{\mathrm{TeV}, \operatorname{Max}}=4.9 \cdot 10^{35} \mathrm{erg} \mathrm{cm}^{-2} \mathrm{~s}^{-1}\left(L_{\mathrm{TeV}, \operatorname{Max}}=6.8 \cdot 10^{35} \mathrm{erg} \mathrm{cm}^{-2} \mathrm{~s}^{-1}\right)$ and $\tau=1.8 \cdot 10^{3} \mathrm{y}\left(\tau=0.5 \cdot 10^{3} \mathrm{y}\right)$ for $\alpha=1.5(\alpha=1.8)$ given in [5] and by varying the flux ratio in the range $500 \leq R_{\Phi} \leq 1000$. Theoretical predictions can be compared with observational results. It should be remarked that, while PWNe provide the prominent contribution of the observed emission at $\mathrm{TeV}$ energies, they are a subdominant component in the $\mathrm{GeV}$ domain. The 3FGL catalog, which is mostly composed by extragalactic objects, includes 11 PWNe, 23 SNRs, and 49 objects (labelled as SPP) of unknown nature but overlapping with known SNRs or PWNe [3]. The magenta line in Fig. 1 corresponds to the distribution of PWNe included in 3FGL while the black line also include SPP sources. The SPP source class is not expected to fully correspond to the population considered in this work; it can be however regarded as an upper limit for theoretical predictions. A reasonable agreement exists with theoretical expectations, supporting the phenomenological description adopted in this paper.

\section{Results}

Faint sources cannot be individually resolved by Fermi-LAT and contribute to the large scale diffuse emission observed by this experiment. The unresolved PWNe contribution can be calculated as:

$$
\Phi_{\mathrm{GeV}}^{\mathrm{NR}}=\int_{0}^{\Phi_{\mathrm{GeV}}^{\mathrm{th}}} d \Phi_{\mathrm{GeV}} \Phi_{\mathrm{GeV}} \frac{d N}{d \Phi_{\mathrm{GeV}}}
$$

where $d N / d \Phi_{\mathrm{GeV}}$ is the source flux distribution in the $\mathrm{GeV}$ domain while $\Phi_{\mathrm{GeV}}^{\mathrm{th}}=10^{-9} \mathrm{~cm}^{-2} \mathrm{~s}^{-1}$ is the Fermi-LAT detection threshold [3]. In the last line of Tab.1, we give the cumulative flux $\Phi_{\mathrm{GeV}}^{\mathrm{NR}}$ 
Table 1: The cumulative flux of resolved $\left(\Phi_{\mathrm{GeV}}^{\mathrm{R}}\right)$ and unresolved $\left(\Phi_{\mathrm{GeV}}^{\mathrm{NR}}\right) \mathrm{TeV} P$ WNe in the GeV domain for $\alpha=1.8$ and for the two different values of $R_{\Phi}$ considered in our analysis. The Fermi-LAT diffuse emission $\Phi_{\mathrm{GeV}}^{\mathrm{diff}}$ is shown in the first column [8]. The numbers in brackets give the ratios $\Phi_{\mathrm{GeV}}^{\mathrm{NR}} / \Phi_{\mathrm{GeV}}^{\mathrm{diff}}$ in different galactocentric rings.

\begin{tabular}{l|c|cc|cc}
\hline \hline Ring (kpc) & $\Phi_{\mathrm{GeV}}^{\mathrm{diff}}\left(\mathrm{cm}^{-2} \mathrm{~s}^{-1}\right)$ & $\Phi_{\mathrm{GeV}}^{\mathrm{NR}}\left(\mathrm{cm}^{-2} \mathrm{~s}^{-1}\right)$ & & $\Phi_{\mathrm{GeV}}^{\mathrm{R}}\left(\mathrm{cm}^{-2} \mathrm{~s}^{-1}\right)$ \\
\hline & & $R_{\Phi}=500$ & $R_{\Phi}=1000$ & $R_{\Phi}=500$ & $R_{\Phi}=1000$ \\
\hline $1.7-4.5$ & $3.86 \times 10^{-7}$ & $6.63 \times 10^{-8}(17 \%)$ & $1.15 \times 10^{-7}(29.9 \%)$ & $2.78 \times 10^{-8}$ & $7.29 \times 10^{-8}$ \\
$4.5-5.5$ & $3.11 \times 10^{-7}$ & $3.8 \times 10^{-8}(12.2 \%)$ & $6.62 \times 10^{-8}(21.2 \%)$ & $2.1 \times 10^{-8}$ & $5.2 \times 10^{-8}$ \\
$5.5-6.5$ & $5.09 \times 10^{-7}$ & $4.24 \times 10^{-8}(8.3 \%)$ & $7.37 \times 10^{-8}(14.4 \%)$ & $3.0 \times 10^{-8}$ & $7.14 \times 10^{-8}$ \\
$6.5-7.0$ & $2.57 \times 10^{-7}$ & $2.28 \times 10^{-8}(8.8 \%)$ & $3.96 \times 10^{-8}(15.3 \%)$ & $2.08 \times 10^{-8}$ & $4.77 \times 10^{-8}$ \\
$7.0-8.0$ & $7.7 \times 10^{-7}$ & $5.29 \times 10^{-8}(6.8 \%)$ & $9.21 \times 10^{-8}(11.9 \%)$ & $7.03 \times 10^{-8}$ & $1.54 \times 10^{-7}$ \\
$8.0-10.0$ & $3.84 \times 10^{-6}$ & $9.69 \times 10^{-8}(2.5 \%)$ & $1.68 \times 10^{-7}(4.3 \%)$ & $2.24 \times 10^{-7}$ & $4.74 \times 10^{-7}$ \\
$10.0-16.5$ & $7.68 \times 10^{-7}$ & $3.0 \times 10^{-8}(3.9 \%)$ & $5.24 \times 10^{-8}(6.8 \%)$ & $1.9 \times 10^{-8}$ & $4.56 \times 10^{-8}$ \\
$16.5-50.0$ & $4.44 \times 10^{-8}$ & $7.73 \times 10^{-10}(1.7 \%)$ & $1.38 \times 10^{-9}(3.1 \%)$ & $9.23 \times 10^{-11}$ & $3.44 \times 10^{-10}$ \\
\hline $0.0-50.0$ & $6.89 \times 10^{-6}$ & $3.55 \times 10^{-7}(5.1 \%)$ & $6.18 \times 10^{-7}(8.9 \%)$ & $4.15 \times 10^{-7}$ & $9.23 \times 10^{-7}$ \\
\hline \hline
\end{tabular}

$\left(\Phi_{\mathrm{GeV}}^{\mathrm{R}}=\Phi_{\mathrm{GeV}}^{\mathrm{tot}}-\Phi_{\mathrm{GeV}}^{\mathrm{NR}}\right)$ produced by TeV PWNe that are not resolved (resolved) by Fermi-LAT for the two assumed values $R_{\Phi}=500$ and 1000. These fluxes are compared with the total large scale diffuse emission $\Phi_{\mathrm{GeV}}^{\mathrm{diff}}$ detected by Fermi-LAT (second column in Tab.1) in the $1-100 \mathrm{GeV}$ energy range [8]. Unresolved emission by PWNe corresponds to a fraction $\sim 5 \%$ (for $R_{\Phi}=500$ ) and $\sim 9 \%$ (for $R_{\Phi}=1000$ ) of the total large scale diffuse emission ${ }^{1}$.

In order to probe the radial dependence of the PWNe contribution, we repeat our calculations by considering the Galactocentric rings adopted by [8]. The flux produced by unresolved $\mathrm{TeV}$ PWNe in each ring is compared with the Fermi-LAT diffuse emission from the same region. As we see from Tab. 1, the unresolved contribution becomes more relevant in the central rings, due the fact that the source density (and the average distance from the Sun position) is larger. In the most internal region $(1.7 \leq r \leq 4.5 \mathrm{kpc})$, unresolved sources account for about $\sim 20 \%(\sim 30 \%)$ of the Fermi-LAT diffuse emission for $R_{\Phi}=500\left(R_{\Phi}=1000\right)$. This clearly shows that this component is not negligible and cannot be ignored in the interpretation of Fermi-LAT diffuse emission data.

The effect of the unresolved TeV PWNe population on the determination of CR diffuse emission is discussed in Fig. 2. Black data points show the total diffuse $\gamma$-ray flux observed by Fermi-LAT in each galactocentric ring given by [8] in $25 \log$-spaced energy bins between $0.34-228.65 \mathrm{GeV}$ and in the latitude window $|b|<20.25^{\circ}$. These data have been fitted with a single power-law $\propto E^{-\Gamma_{1}}$ by [8], obtaining the green dashed lines reported in Fig.2. The decrease of the spectral indexes $\Gamma_{1}$ in the inner rings with respect to the local value, see Tab.2, has been considered as the evidence of a progressive large-scale hardening of CRs spectrum toward the Galactic Center (see also [4, 12]).

The above conclusion is only valid if unresolved source contribution is negligible, so that the total observed emission can be identified with the "truly" diffuse component produced by CR interaction with interstellar matter. This assumption is, however, not adequate in the inner Galaxy, as it is shown by the red solid lines in Fig.2 that give the unresolved PWNe contribution as function of energy for $R_{\Phi}=500$ and 1000. In order to quantify the effect of unresolved PWNe, we perform a new fit of the Fermi-LAT data. The truly diffuse gamma-ray flux due to CR interactions is still

\footnotetext{
${ }^{1}$ The above predictions are obtained by assuming that the source luminosity distribution index is $\alpha=1.8$ to conform with previous analyses $[4,8]$. Results for $\alpha=1.5$ are reported as additional material in Ref.[11]
} 
parameterized as a single power-law (the number of degrees of freedom in the fit is not changed) but the total flux, described with blue lines in Fig.2, is obtained as the sum of CR diffuse emission plus the unresolved PWNe contribution. The best-fits spectral indexes $\Gamma_{B F}$ for CR diffuse emission in each ring are reported in Tab.2 for $R_{\Phi}=500$ and 1000. These correspond to the thick solid green lines reported in Fig.2. The obtained values for $\Gamma_{B F}$ are mildly dependent on the assumed $R_{\Phi}$.

It is evident that the unresolved PWNe contribution affects the reconstructed properties of CR diffuse emission, weakening considerably the evidence of CR spectral hardening in the central region of the Galaxy. In order to quantify this point, we show in Fig.3 the difference $\Delta \Gamma=\Gamma_{B F}-\Gamma_{1}$ where $\Gamma_{1}$ is the best-fit for the truly diffuse emission obtained by [8] while $\Gamma_{B F}$ is the best-fit for the same quantity obtained in this work. The reported error bar takes also into account the effect of possible variations of the parameter $R_{\Phi}$ within the range $R_{\Phi}=500-1000$. The inclusion of unresolved PWNe strongly affects the spectral index of CR diffuse emission that can be increased up to $\Delta \Gamma=0.17$ in the central ring adjusting it to the locally observed value, i.e. $\sim 2.7$. The evidence of spectral hardening toward the Galactic Center is reduced.

Table 2: Spectral indexes of the CR diffuse emission obtained by fitting the Fermi-LAT data with $\left(\Gamma_{B F}\right)$ and without $\left(\Gamma_{1}\right) \mathrm{TeV} P W N e$ unresolved contribution. The indexes $\Gamma_{1}$ coincide with those obtained by [8].

\begin{tabular}{l|c|cc}
\hline \hline Ring & $\Gamma_{1}$ & $\begin{array}{c}\Gamma_{B F} \\
R_{\Phi}=500\end{array}$ & $\begin{array}{c}\alpha=1.8 \\
R_{\Phi}=1000\end{array}$ \\
\hline $1.7-4.5 \mathrm{kpc}$ & $2.56 \pm 0.02$ & $2.72 \pm 0.01$ & $2.72 \pm 0.01$ \\
$4.5-5.5 \mathrm{kpc}$ & $2.48 \pm 0.02$ & $2.57 \pm 0.01$ & $2.56 \pm 0.01$ \\
$5.5-6.5 \mathrm{kpc}$ & $2.54 \pm 0.04$ & $2.63 \pm 0.01$ & $2.63 \pm 0.01$ \\
$6.5-7 \mathrm{kpc}$ & $2.54 \pm 0.01$ & $2.62 \pm 0.01$ & $2.61 \pm 0.02$ \\
$7-8 \mathrm{kpc}$ & $2.57 \pm 0.01$ & $2.625 \pm 0.008$ & $2.623 \pm 0.008$ \\
$8-10 \mathrm{kpc}$ & $2.642 \pm 0.003$ & $2.663 \pm 0.003$ & $2.662 \pm 0.004$ \\
$10-16.5 \mathrm{kpc}$ & $2.696 \pm 0.008$ & $2.743 \pm 0.008$ & $2.740 \pm 0.009$ \\
$16.5-50 \mathrm{kpc}$ & $2.72 \pm 0.03$ & $2.77 \pm 0.04$ & $2.76 \pm 0.03$ \\
\hline \hline
\end{tabular}

\section{Conclusions}

The TeV Galactic sky is dominated by a population of bright young PWNe whose properties are constrained by present HGPS data. We predict the cumulative emission produced by this population in the $\mathrm{GeV}$ domain within a phenomenological model based on the average spectral properties of PWNe. We show that a relevant fraction of the TeV PWNe emission cannot be resolved by FermiLAT and adds up to CR diffuse flux, shaping the radial and spectral behaviour of the total diffuse $\gamma$-ray emission observed by Fermi-LAT. The emergence of PWNe unresolved component in the central region of the Galaxy can strongly affect the interpretation of Fermi-LAT data by naturally accounting for (a large part of) the observed diffuse flux spectral index variation.

\section{Acknowledgments}

This work was partially supported by the research grant number 2017W4HA7S "NAT-NET: Neutrino and Astroparticle Theory Network" under the program PRIN 2017 funded by the Italian Ministero dell'Istruzione, dell'Universita' e della Ricerca (MIUR). 

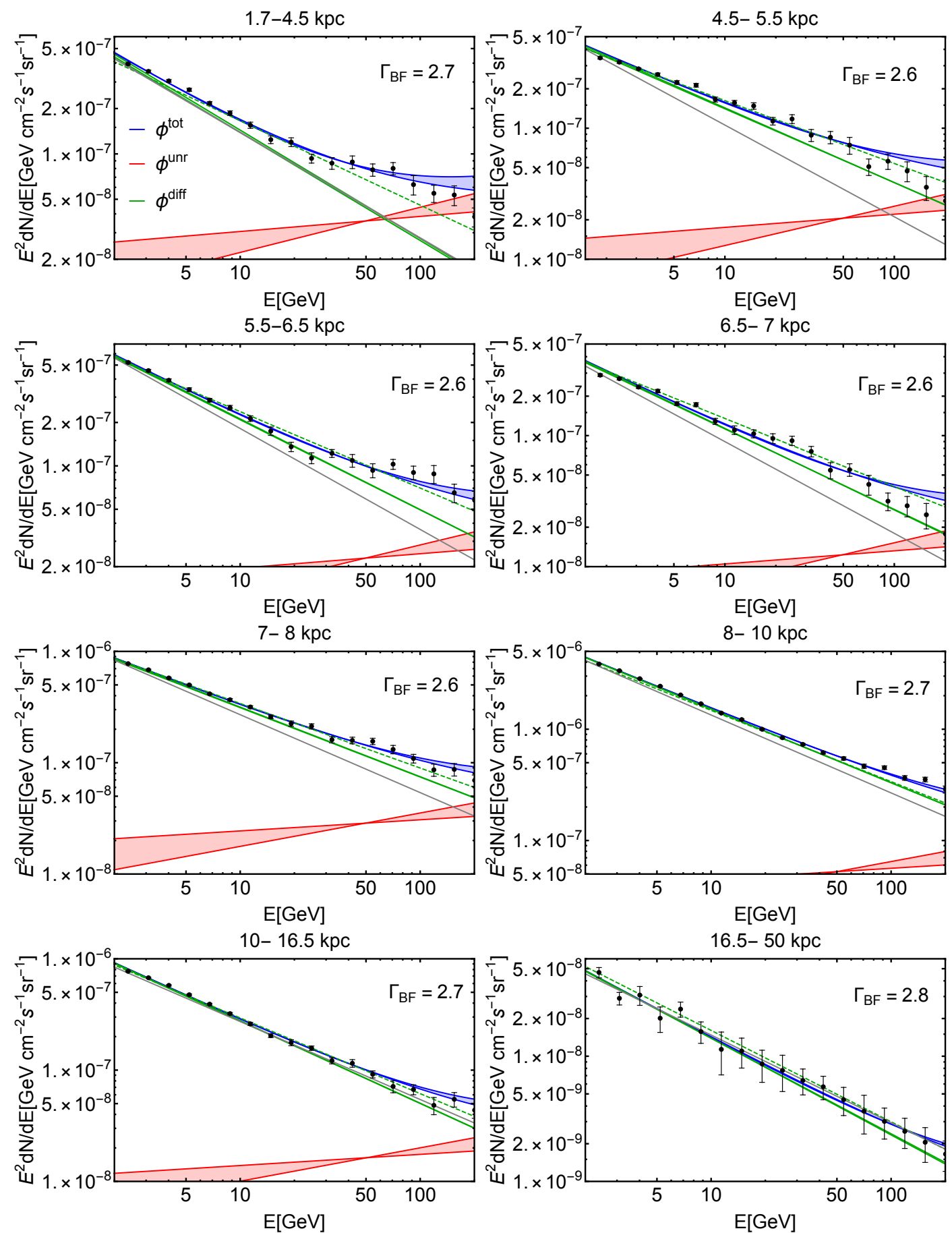

Figure 2: Black data points show the total diffuse $\gamma$-ray emission measured by Fermi-LAT in each galactocentric ring [8]. The red lines represent the predicted contribution of unresolved TeV PWNe for $\alpha=1.8$. Green lines show the diffuse CR emission inferred by fitting the data with (solid) and without (dashed) including the PWNe contribution. Blue lines represent the total gamma fluxes predicted as a function of the energy for $\alpha=1.8$. The gray lines show a power-law with an index of 2.7 for comparison. 


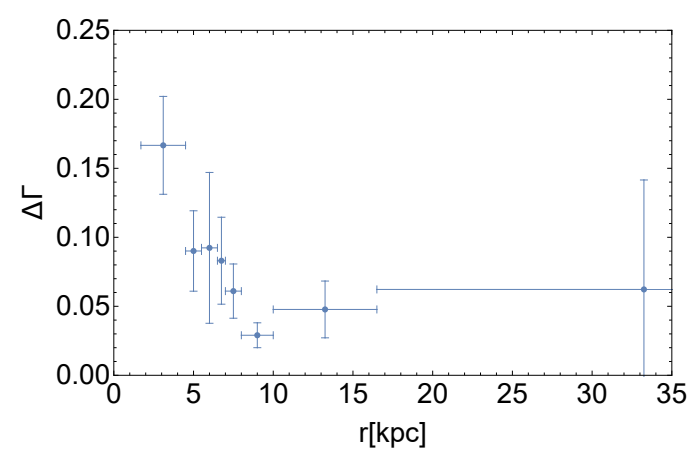

Figure 3: The difference $\Delta \Gamma$ between the spectral index of the truly diffuse emission obtained in different Galactocentric rings by fitting the Fermi-LAT data with/without the contribution of unresolved PWNe.

\section{References}

[1] Abdalla, H., Abramowski, A., Aharonian, F., et al. 2018, Astron. Astrophys., 612, A2, doi: 10. 1051/0004-6361/201629377

[2] —. 2018, Astron. Astrophys., 612, A1, doi: 10.1051/0004-6361/201732098

[3] Acero, F., M. Ackermann(DESY), M., Ajello, Albert, A., et al. 2015, Astrophys. J. Suppl., 218, 23, doi: $10.1088 / 0067-0049 / 218 / 2 / 23$

[4] Acero, F., Ackermann, M., Ajello, M., et al. 2016, Astrophys. J. Suppl., 223, 26, doi: 10 . 3847 / 0067-0049/223/2/26

[5] Cataldo, M., Pagliaroli, G., Vecchiotti, V., \& Villante, F. L. 2020, Astrophys. J., 904, 85, doi: $10.3847 / 1538-4357 /$ abc0ee

[6] Diehl, R., Halloin, H., Kretschmer, K., et al. 2006, Nature, 439, 45, doi: 10.1038/ nature 04364

[7] Lorimer, D. R., Faulkner, A. J., Lyne, A. G., et al. 2006, Mon. Not. Roy. Astron. Soc., 372, 777, doi: $10.1111 / j .1365-2966.2006 .10887 . x$

[8] Pothast, M., Gaggero, D., Storm, E., \& Weniger, C. 2018, JCAP, 10, 045, doi: 10.1088/ $1475-7516 / 2018 / 10 / 045$

[9] Sudoh, T., Linden, T., \& Beacom, J. F. 2019, Phys. Rev. D, 100, 043016, doi: 10.1103/ PhysRevD. 100.043016

[10] Torres, D. F., Cillis, A., Martín, J., \& de Oña Wilhelmi, E. 2014, JHEAp, 1-2, 31, doi: 10. 1016/j . jheap. 2014.02.001

[11] Vecchiotti, V., Pagliaroli, G., \& Villante, F. L. 2021, doi: 10.21203/rs . 3.rs-539249/v1

[12] Yang, R., Aharonian, F., \& Evoli, C. 2016, Phys. Rev. D, 93, 123007, doi: 10.1103/ PhysRevD. 93.123007 